\title{
Uma Análise Comparativa de Kits para a Robótica Educacional
}

\author{
Almir de Oliveira Costa Jr., Elloá B. Guedes \\ ${ }^{1}$ Escola Superior de Tecnologia \\ Universidade do Estado do Amazonas \\ Av. Darcy Vargas, 1200 - Manaus - Amazonas \\ \{almirlinux, elloa.uea\}@gmail.com
}

\begin{abstract}
Which characteristics are relevant and must be considered when choosing a educational robotics kit? Kits for educational robotics are, in their essence, composed by hardware, software and pedagogic resources aiming at easing its adoption in classrooms. However, we have to consider the different demands of each context as well as the different characteristics of each kit. Taking that into account, this work presents and analises three educational robotics kits under many aspects, such as hardware finishing, available pedagogic resources, among others. Besides emphasizing and contrasting the kits, other contribution of this paper is to provide information in the decision making process of which kit must be considered in real classrooms contexts.
\end{abstract}

Resumo. Quais aspectos são relevantes e precisam ser considerados na escolha de um kit de robótica educacional? Kits de robótica educacional, em sua essência, proveem hardware, software e material pedagógico para viabilizar a utilização deste recurso no contexto da sala de aula, mas há que se considerar as necessidades de cada contexto e as características diferentes que cada kit possui. Levando isto em consideração, este trabalho apresenta e analisa comparativamente as características de três kits para robótica educacional disponíveis no mercado, considerando diferentes aspectos nesta análise, tais como acabamento do hardware, material pedagógico disponível, dentre outros. Além de enfatizar e contrastar as características dos kits apresentados, outra contribuição deste artigo é fornecer informações para o processo de tomada de decisão na escolha dos kits de robótica a serem adotados em contextos reais de sala de aula.

\section{Introdução}

Desde a última década a robótica tem atraído o interesse de professores e pesquisadores por se caracterizar como uma ferramenta importante para o desenvolvimento cognitivo e das habilidades sociais de estudantes de diversos níveis [Alimisis 2013]. De acordo com Silva et al. [Silva et al. 2009], isto é resultado de mudanças significativas no âmbito social, cuja a principal característica seria a modificação de uma cultura material para um novo paradigma tecnológico organizado em torno de tecnologias da informação.

Em virtude da crescente importância da robótica e das vantagens identificadas na sua adoção em escolas, cunhou-se a Robótica Educacional, ou Robótica Pedagógica, que consiste basicamente na aprendizagem por meio de montagem de sistemas constituídos por modelos. Esses modelos são robôs, os quais consistem de diferentes mecanismos e que realizam atividades de natureza física, tais como, movimento de um braço mecânico, 
levantamento de objetos, etc. [Bacaroglo 2005]. A robótica educacional tem sido considerada importante para o ensino das ciências, da matemática, tecnologia, informática, outras matérias e também de atividades interdisciplinares [Alimisis 2013].

Considerando a importância e a necessidade das escolas incluírem a Robótica Educacional, muitas empresas tem comercializado kits de robótica educacional, que incluem hardware, software e material pedagógico para integrar este recurso na formação dos estudantes. Estes kits possuem características diferentes e que precisam ser consideradas na escolha do kit mais adequado para um determinado contexto. Porém, há poucas referências que sintetizem as características de cada kit, com o intuito de analisá-los e contrastá-los. Levando em consideração esta carência da literatura, este artigo apresenta três kits de robótica educacional amplamente conhecidos e efetua uma análise comparativa dos mesmos, considerando 12 critérios diferentes, tais como demanda de conhecimentos prévios, acabamento do hardware, características da linguagem de programação utilizada, dentre outros.

Para apresentar estes resultados, o artigo está organizado como segue. A robótica educacional e os conceitos relacionados a ela encontram-se apresentados na Seção 2. Os kits considerados no escopo deste trabalho e uma visão geral de suas características encontram-se descritos na Seção 3. A análise comparativa é apresentada e discutida na Seção 4. Por fim, as considerações finais são mostradas na Seção 5.

\section{Robótica Educacional}

Nos dias atuais, muitos professores tem visto na robótica um recurso tecnológico bastante interessante e rico para intervir no processo de ensino-aprendizagem, pois ela contempla o desenvolvimento pleno do aluno por meio de atividades dinâmicas [Zilli 2004]. A utilização da robótica neste contexto pode focar-se na montagem de dispositivos, programação e trabalhar conceitos ligados a matérias curriculares, tais como Física e Matemática [da Silva and Scherer 2013].

A Robótica Educacional, ou Robótica Pedagógica, pode ser entendida como o ambiente de aprendizagem no qual o professor ensina ao aluno a montagem, automação e controle de dispositivos mecânicos que podem ser controlados por um computador [César 2005]. O precursor desta área foi Seymour Papert, que via no computador e suas possibilidades um recurso que atraía as crianças e, com isso, facilitava o processo de aprendizagem. Este pesquisador concebeu uma linguagem de programação para crianças e que, posteriormente, em conjunto com o Instituto de Tecnologia de Massachusetts (MIT), vislumbrou a possibilidade das crianças concretizarem seus protótipos utilizando brinquedos (tijolinhos, motores, engrenagens, polias e sensores) de forma a externalizar comportamentos aos protótipos montados [Papert 1994].

Para facilitar a utilização da robótica no contexto da sala de aula, kits de robótica educacional são normalmente utilizados. Estes kits são compostos basicamente por hardware, software e documentação. No tocante ao hardware, os componentes frequentemente encontrados são:

1. Unidade programável. Consiste dos microcontroladores que irão receber os programas construídos pelos estudantes;

2. Componentes estruturais. São compostos por manipuladores, rodas, carcaça, dentre outros.

3. Componentes eletrônicos. Permitem a adição de recursos de movimento, tais como motores, e também a interação do robô com o meio, tais como dispositivos 
de som, sensores, dentre outros [da Silva and Scherer 2013].

Em relação ao software, há normalmente dois tipos de linguagens de programação associadas a um kit de robótica. O primeiro tipo é uma linguagem de programação textual, podendo ser desenvolvida para o próprio kit, ser inspirada em uma linguagem de programação amplamente divulgada (como C ou Java, por exemplo) ou consistir de uma biblioteca para uma linguagem de programação. O segundo tipo é uma linguagem de programação gráfica, ou seja, cujos programas são construídos a partir da interação do usuário com elementos visuais e por meio de ações com o mouse, principalmente. Estas linguagens são ricas em ícones, cores, símbolos, imagens e afins, para facilitar a interação com o usuário, que são principalmente crianças.

A documentação, por sua vez, consiste de: (i) manual do usuário, no qual é mostrada a relação das peças disponíveis no kit e as instruções para instalação e utilização do software; (ii) documentação técnica, que abrange dados mais detalhados, com o intuito de permitir a construção ou utilização componentes extras; e (iii) material de apoio pedagógico, no qual há instruções para a utilização do kit junto aos estudantes, incluindo, especialmente, tipos diferentes de projetos que podem ser desenvolvidos com cada kit [da Silva and Scherer 2013].

Considerando os atributos gerais de kits de robótica, a próxima seção apresenta alguns destes kits, cujas características serão detalhadas.

\section{Kits para o Ensino de Robótica Educacional}

Conforme apresentado na seção anterior, os kits de robótica são produtos comercializados por determinadas empresas com o intuito de facilitar a adoção da Robótica Educacional nas escolas. Nesta seção, três modelos de kits de robótica são apresentados em detalhes: o Lego Mindstorm, o Modelix e o Arduino. A escolha por detalhar esses três modelos levou em consideração os seguintes aspectos: o fato de serem os mais populares; possuírem linguagem de programação gráfica e também por possuírem documentação em língua portuguesa, facilitando a adoção por escolas lusófonas.

\subsection{Lego Mindstorm}

O kit Lego Mindstorm, comercializado pela empresa dinamarquesa Lego, teve sua produção iniciada em 1999 e contém software e hardware que permitem a criação de robôs customizáveis e programados [Lego 2015]. Atualmente, em sua terceira geração, denominada Lego Mindstorms EV3, este kit inclui componentes eletrônicos e estruturais, o software EV3, instruções de montagem e um circuito de testes. Em particular, a unidade programável deste kit chama-se EV3 Brick, que além do microprocessador, inclui 4 portas de entrada, 4 portas de saída e entrada para cartão de memória [Park 2014].

A caixa na qual o Lego Mindstorm EV3 é comercializado encontra-se ilustrada na Figura 1a. O EV3 Brick pode ser visualizado na Figura 1b. Por fim, o circuito de testes é mostrada na Figura 1c. Este circuito é uma espécie de "pista" na qual o robô pode se deslocar e testar a maioria dos seus sensores e motores 


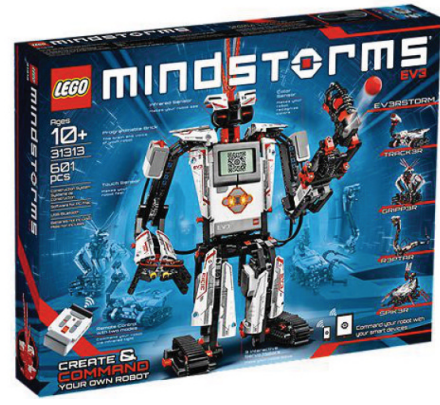

(a) Lego Mindstorm EV3

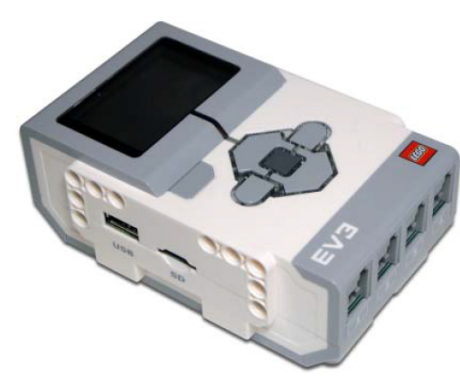

(b) EV3 Brick

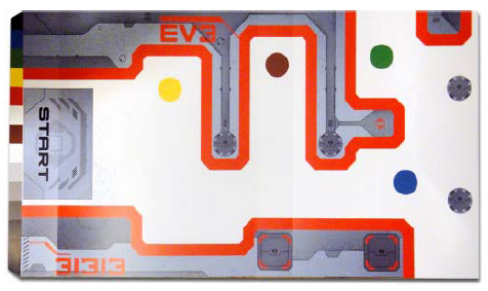

(c) Circuito

Figura 1: Ilustrações do kit de robótica Lego Mindstorm EV3. Fonte: [Park 2014].

Na sua versão atual, o kit permite que os robôs sejam controlados por dispositivos inteligentes, tais como smartphones ou tablets. Além disso, conexão Bluetooth, sensor de toque e controle remoto [Park 2014].

A programação dos robôs Lego Mindstorm EV3 é feita com a linguagem de programação gráfica Mindstorms EV3 programming software, que pode ser obtido no site oficial da empresa Lego [Lego 2015]. A programação do robô é feita de maneira visual, na qual blocos de funcionalidades são agrupados tais como peças de um quebra cabeça, permitindo a execução de diversos tipos de comandos, que vão desde a movimentação do robô até a leitura de dados dos sensores.

Há cinco tipos de blocos disponíveis na linguagem de programação dos robôs Lego Mindstorm EV3: blocos de ação, blocos de fluxo, blocos de sensores, blocos de operações de dados e, por último, os blocos avançados. Estes últimos permitem a realização de atividades mais complexas, tais como a manipulação de arquivos, conexões Bluetooth, dentre outros. Uma combinação dos blocos para a construção de um programa é mostrada na Figura ??. Neste programa, há um bloco de controle que marca o início do programa, um bloco de sensor para recebimento de dados do sensor ultrassônico, um bloco de ação, responsável por escrever o valor obtido pelo sensor no display do EV3 brick e um bloco de controle responsável por repetir as duas últimas ações continuamente, por meio de um laço infinito.

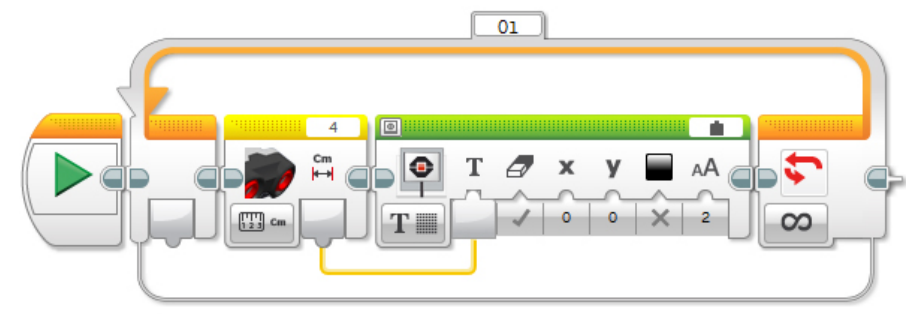

Figura 2: Exemplo de programa construído para o Lego Mindstorm EV3. Fonte: [Afrel 2015].

\subsection{Modelix Robotics}

O kit Modelix Robotics visa o desenvolvimento de projetos de robótica para diferentes níveis educacionais. Em sua versão 3.6, o kit é composto por microcontrolador, display LCD, joystick, controle remoto, fonte de alimentação e diversos outros componentes, tais como sensores, atuadores e componentes estruturais [Modelix 2015b]. 
Para programação, o Modelix System Pro é o software disponível que possui dois modos de utilização:

1. Modo Programação. Neste modo, o estudante irá construir fluxogramas que descrevem o que o robô deve fazer, permitindo o controle de motores, sensores, luzes, dentre outros. Segundo o fabricante, há suporte para a detecção de erros. Após esta etapa, o programa pode ser transferido e executado pelo robô. A Figura 3a ilustra um exemplo de fluxograma construído neste modo;

2. Modo de Simulação de Cenários. Simula ambientes reais como, por exemplo, a casa automática ou um trem de passageiros que será controlado por uma rotina de programação feita pelo aluno. Neste modo, o cenário é simulado virtualmente, dispensando o uso de robôs ou outros componentes físicos. A Figura $3 b$ ilustra um exemplo de cenário simulado.

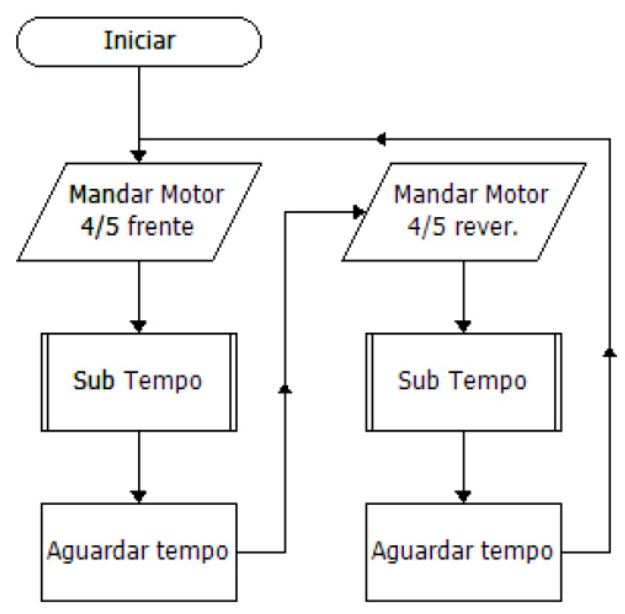

(a) Modo Programação

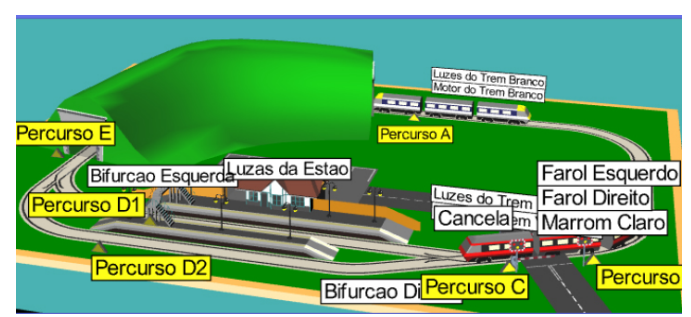

(b) Modo Simulação

Figura 3: Ilustrações dos modos de programação dos robôs Modelix. Fonte: [Modelix 2015a].

\subsection{Arduino}

O Projeto Arduino, foi criado na Itália em 2005 com o objetivo de oferecer uma plataforma de prototipagem eletrônica, sob a forma de uma placa eletrônica programável, de baixo custo e de fácil manuseio. O Arduino enquadra-se como um projeto de hardware open source, no qual a documentação para a confecção da placa eletrônica é livremente disponibilizado, facilitando a sua produção e também permitindo que os usuários possam conhecer melhor o hardware, utilizando este conhecimento para o desenvolvimento de diversos projetos [Blum 2013].

De acordo com da Silva e Scherer, a plataforma Arduino não foi concebida para robótica educacional, porém há alguns kits comercializados para este propósito que permitem o desenvolvimento de atividades com robótica, mas que exigem algum conhecimento de eletrônica básica [da Silva and Scherer 2013]. Para a construção de robôs com Arduino, há três elementos essenciais:

1. A placa Arduino. É a placa em si. A depender do modelo, consiste de um microcontrolador, interfaces USB, regulador de voltagem, conector de energia, pinos de entrada e saída, botão de reset, conectores para programação no circuito e LEDs para indicar algumas funções (debug e funcionamento, por exemplo). Um dos modelos de placa Arduino, o Arduino Uno, é ilustrado na Figura 4a; 
2. Hardware externo. Inclui a carcaça, circuitos feitos à mão, e hardware construído por terceiros, tais como display, rodas, motores, dentre outros;

3. Arduino IDE. É o ambiente de desenvolvimento integrado, compatível com Windows e Linux. A linguagem de programação oficial do Arduino é baseada na linguagem $\mathrm{C} / \mathrm{C}++$, porém com algumas particularidades para geração automática de protótipos de funções [Arduino 2015].

Embora o Arduino IDE seja uma linguagem de programação para esta plataforma, no escopo deste trabalho será considerado o Scratch for Arduino (S4A), uma modificação na linguagem de programação gráfica Scratch que permite a programação do Arduino de maneira simplificada, permitindo a utilização dos sensores e atuadores conectados à placa, e, por conseguinte, a construção de robôs [S4A 2015]. O ambiente S4A encontrase ilustrado na Figura $4 b$.

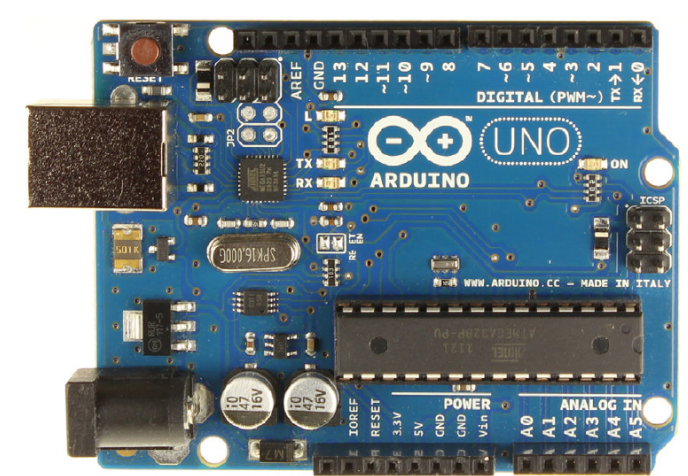

(a) Arduino Uno

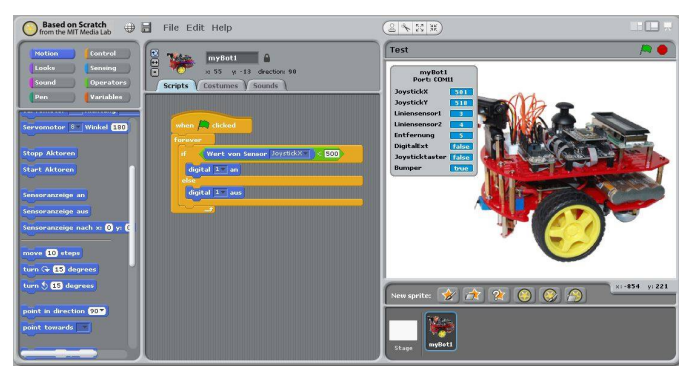

(b) S4A

Figura 4: Ilustrações dos modos de programação dos robôs Modelix.

\section{Análise Comparativa}

Como visto na seção anterior, há diferentes kits de robótica educacional com características diferentes. A escolha de um determinado kit para adoção em certo contexto educacional precisa levar em conta estas diferenças, com o intuito de identificar o qual melhor atende às necessidades desse contexto. Em virtude disso, com o intuito de analisar comparativamente os kits apresentados, serão considerados diferentes aspectos, que incluem desde a avaliação do material pedagógico até o custo destes kits.

Em termos de material pedagógico, destacam-se os kits Lego Mindstorm e Modelix Robotics. O primeiro kit possui a documentação em português Lego Zoom, que consiste em um conjunto de revistas organizadas de acordo com o nível de ensino, desde a pré-escola até o ensino médio [Lego Zoom 2015]. Estas revistas possuem projetos de robótica educacional adequados para cada nível, que mostram detalhadamente desde a montagem dos robôs até a construção do programa que estes devem executar. O kit Modelix Robotics, por sua vez, possui também um material pedagógico que contém planos de aula, projetos de robótica, manuais e tutoriais diversos [Modelix 2015a]. O número de projetos sugerido pelo material pedagógico é mais limitado quando comparado ao kit anterior. Um outro aspecto que poderia ser melhorado é a sugestão de projetos por níveis educacionais, que não é feita neste material, apenas há a indicação de que é adequado para diferentes níveis. Por fim, não foram encontrados materiais pedagógicos para os kits implementados com Arduino. 
No que se refere ao acabamento do hardware, os kits da Modelix Robotics e do Arduino são bastante semelhantes. Ambos se baseiam em uma unidade programável sob a forma de placa e em componentes que são acrescidos à ela. Para estes dois kits, há a necessidade de um certo conhecimento em eletrônica para construção dos robôs e a manipulação de ferramentas como chave de fenda e de rosca se faz necessária. Este é uma exigência que pode dificultar a adoção desses kits com crianças pequenas. O kit Lego Mindstorm não demanda conhecimentos em eletrônica e a utilização de ferramentas é dispensada, pois todas as montagens podem ser feitas utilizando apenas as mãos.

A extensibilidade do hardware, isto é, a capacidade de serem acrescidos novos componentes, é um aspecto que ressalta algumas diferenças entre os kits. Os kits com maior extensibilidade são o Arduino e o Modelix, pois a cada dia surgem novos sensores e atuadores, inclusive produzidos por outros fabricantes. No caso do kit Lego Mindstorm, a possibilidade de acrescentar sensores e atuadores também é verificada. Porém, é necessário que sejam produzidos e disponibilizados pelo próprio fabricante.

Em se tratando da possibilidade de construir novo hardware, os kits Modelix e Arduino permitem que os estudantes possam construir seus próprios componentes, utilizando inclusive sucata, por exemplo. Isto não é possível com o kit Lego Mindstorm, pois as informações sobre voltagem e padrões de comunicação entre os sensores e atuadores, por exemplo, são de tecnologia proprietária, cuja documentação não é amplamente disponível.

Embora no aspecto anterior os kits Modelix e Arduino se destaquem, por este mesmo aspecto eles possuem uma avaliação negativa no tocante ao conhecimento prévio requerido para sua utilização. Estes dois kits demandam conhecimentos prévios de eletrônica para permitir a construção de robôs, mesmos os mais elementares. Esta demanda é necessária tanto para o professor que irá ministrar as aulas de robótica quanto para os estudantes que irão utilizar os kits. No kit Lego Mindstorm nenhum conhecimento prévio desta natureza é necessário para sua utilização. Vale salientar, entretanto, que a demanda por conhecimentos de eletrônica pode ser aproveitada de maneira positiva em disciplinas voltadas para o ensino técnico.

Em termos da linguagem de programação gráfica utilizada pelos kits, há diferentes aspectos a serem considerados. O kit Modelix incorpora a notação de fluxogramas, já os kits Lego Mindstorm e Arduino incorporam a ideia de blocos. Embora a notação de fluxogramas seja amplamente conhecida no domínio da programação, ela precisa ser ensinada às crianças, enquanto os blocos e suas combinações são mais intuitivos, pois as crianças já possuem brinquedos que trazem esta ideia. A utilização de cores, por sua vez, pode chamar a atenção de diferentes aspectos, separando os tipos de comandos ou mostrando o fluxo de execução, por exemplo. Este recurso é utilizado no Lego Mindstorm e no Arduino, mas não no kit Modelix.

A usabilidade da interface de programação, isto é, a facilidade de utilizar a linguagem de programação gráfica também é um aspecto a ser avaliado. A interface considerada de mais fácil utilização na avaliação efetuada foi a do Arduino com S4A, pois além de mostrar a programação em si, exibe também dados dos sensores e atuadores em tempo real, permitindo uma melhor analogia entre programação e execução no robô. O kit Lego Mindstorm também possui uma boa usabilidade, pois embora não exiba o robô físico, os seus blocos contem imagens dos sensores e atuadores, facilitando o entendimento do fluxo da informação. O kit Modelix, por sua vez, deixa a desejar neste aspecto, pois 
faltam imagens, cores e outros recursos que tornem a sua utilização mais intuitiva.

A portabilidade dos programas produzidos é considerada muito boa no Arduino, pois podem ser transferidos para outros robôs que usem placas de modelos diferentes. No kit Modelix a portabilidade para outras plataformas não é verificada. No kit Lego Mindstorm só há retrocompatibilidade, ou seja, o hardware mais antigo pode executar comandos produzidos com software mais recente. Porém, esta compatibilidade é restrita apenas para a mesma plataforma.

O ambiente de programação Arduino é o que é mais compatível com diferentes sistemas operacionais, incluindo Linux, Windows, Mac e Raspbian, uma distribuição de Linux que executa no RaspberryPi. O Lego Mindstorm é compatível com Windows e Mac. Já o Modelix é compatível com Windows, Mac e Linux, mas nem todas as funcionalidades estão disponíveis em todos estes sistemas, como é o caso da síntese de voz. A compatibilidade com diferentes sistemas operacionais é importante, pois permite que o ambiente de programação do kit possa ser instalado em um laboratório de informática já existente na escola sem maiores alterações.

Ao se considerar a possibilidade de programar os robôs com uma linguagem de programação textual e já existente, o kit Lego Mindstorm é o que possui mais destaque, pois é compatível com diversas linguagens, a exemplo de Java, C, Python, Fortran, e muitas outras. O Modelix permite apenas o desenvolvimento de programas em sua linguagem gráfica, não havendo informações sobre a utilização de outras linguagens de programação no kit. O Arduino permite a programação em $\mathrm{C} \mathrm{e} \mathrm{C}++$, linguagem nativa desta plataforma.

Em termos de custo, será considerada nesta análise os valores para a aquisição de um robô avulso de cada kit. Este custo não envolve a aquisição do material pedagógico associado, pois, para tanto, há possibilidades de aquisição de pacotes ou planos junto às empresas que comercializam estes produtos. O kit Lego Mindstorms custa atualmente 399, 00 dólares, enquanto um robô do kit Modelix custa em torno de 800, 00 reais. O kit Praxedes na plataforma Arduino custa em torno de 225, 00 reais [da Silva and Scherer 2013]. Embora o custo do primeiro kit seja considerado o mais alto, ele permite diversas montagens, enquanto os demais permitem um número limitado de montagens. Relacionando estas características com um custo-benefício, é possível, portanto, afirmar que esta característica é muito boa nos três kits, pois o custo financeiro é proporcional ao número de montagens possíveis.

A Tabela 1 é útil para sintetizar os aspectos considerados na análise comparativa apresentada. É importante salientar que para cada aspecto resumido de maneira breve, há uma argumentação apresentada anteriormente que o justifica.

\section{Considerações Finais}

Uma das preocupações ao adotar kits para o ensino de robótica educacional na escola diz respeito a qual kit será utilizado, pois há diferentes produtos no mercado com diferentes características. Na tentativa de auxiliar neste processo de escolha, o artigo em questão apresentou e caracterizou três kits de robótica educacional comercializados atualmente. Após esta apresentação, foi feita uma análise comparativa considerando doze aspectos diferentes, tais como a qualidade do material pedagógico, o acabamento do hardware, a linguagem de programação utilizada, dentre outros.

Após esta análise, foi verificado que os kits se assemelham quanto a alguns aspectos, mas que se diferenciam em termos de outros. Por esta razão, não é possível ser taxativo quanto a um único tipo de kit apresentado ser considerado mais adequado que 
Tabela 1: Quadro comparativos com os diferentes aspectos considerados em cada kit.

\begin{tabular}{|c|c|c|c|}
\hline & Lego Mindstorm & Modelix Robotics & Arduino \\
\hline Material Pedagógico & Excelente & Muito bom & Ausente \\
\hline Acabamento do Hardware & Excelente & Ruim & Ruim \\
\hline Extensibilidade do Hardware & Média & Excelente & Excelente \\
\hline Construção de Hardware & Ruim & Muito bom & Muito bom \\
\hline Conhecimentos Prévios & Nenhum & Eletrônica & Eletrônica \\
\hline Notação & Blocos & Fluxograma & Blocos \\
\hline Cores & Sim & Não & Sim \\
\hline Usabilidade & Muito boa & Ruim & Excelente \\
\hline Portabilidade de Programas & Média & Ausente & Muito boa \\
\hline Sistemas Operacionais & Mac e Windows & $\begin{array}{l}\text { Windows, Linux e } \\
\text { Mac }\end{array}$ & $\begin{array}{l}\text { Mac, Windows, Li- } \\
\text { nux e Raspbian }\end{array}$ \\
\hline Linguagens & Diversas & Nenhuma & $\mathrm{C} / \mathrm{C}++$ \\
\hline \multicolumn{4}{|l|}{ Programação Compatíveis } \\
\hline Custo-Benefício & Muito bom & Muito bom & Muito bom \\
\hline
\end{tabular}

os outros. Por exemplo, se os kits Lego Mindstorm e Arduino com a linguagem S4A mostram-se mais adequados para serem programados por crianças, o kit Modelix pode ser interessante para introduzir uma linguagem de programação textual, pois sua linguagem de programação gráfica baseia-se em fluxogramas, comumente adotada como uma etapa preliminar no ensino de programação.

Embora os kits Modelix e Lego Mindstorm já se mostrem como dois kits comercializados para o ensino de robótica contendo um bom acabamento neste sentido, o Arduino ainda é uma plataforma que se mostra em ascensão neste domínio. Como mencionado, ela não é voltada para este fim, mas graças às suas características e o baixo custo associado, pode ser aproveitada em diversos contextos para o ensino de robótica educacional. Para favorecer este crescimento, é necessário um esforço de toda a comunidade acadêmica de Informática na Educação no sentido de conceber material pedagógico para esta plataforma, especialmente no que diz respeito aos projetos que podem ser desenvolvidos com ela.

Como sugestões de trabalhos futuros, almeja-se uma expansão do número de kits de robóticas considerados na análise comparativa. Além disso, sugere-se que a integração da robótica educacional com disciplinas regulares do currículo seja mais profundamente estudada, tendo em vista não somente como isto pode ser feito, mas também considerando o impacto desta integração.

\section{Agradecimentos}

Este trabalho foi desenvolvido com o apoio da Fundação de Amparo à Pesquisa do Estado do Amazonas (FAPEAM).

\section{Referências}

Afrel (2015). EV3 ultrasonic sensor. http://www.afrel.co.jp/en/ archives / 844 . Acessado em 15 de junho de 2015.

Alimisis, D. (2013). Educational robotics: Open questions and new challenges. Themes in Science \& Technology Education, 6(1):63-71.

Arduino (2015). Projeto Arduino. http: / / arduino. cc. Acessado em 15 de junho de 2015. 
Bacaroglo, M. (2005). Robótica educacional: Uma metodologia educacional. Master's thesis, Universidade Estadual de Londrina, Londrina, Paraná.

Blum, J. (2013). Exploring Arduino - Tools and Techniques for Engineering Wizardry. Wiley.

César, D. R. (2005). Robótica livre: Robótica educacional com tecnologias livres. In Fórum Internacional de Software Livre.

da Silva, F. I. and Scherer, D. (2013). Praxedes: Protótipo de um kit educacional de robótica baseado na plataforma Arduino. Revista EAD \& Tecnologias Digitais na Educação, 1(1).

Lego (2015). Lego Mindstorms. http://mindstorms. lego.com. Acessado em 15 de junho de 2015 .

Lego Zoom (2015). Zoom education for life. http: / / zoom. education/. Acessado em 15 de junho de 2015.

Modelix (2015a). Manual do usuário. http://modelix.cc/. Acessado em 15 de junho de 2015.

Modelix (2015b). Modelix robotics. http://modelix.cc/. Acessado em 15 de junho de 2015.

Papert, S. M. (1994). A Máquina das Crianças: Repensando a Escola na Era da Informática. Artes Médicas.

Park, E. J. (2014). Exploring Lego Mindstorms EV3 - Tools and Techniques for Building and Programming Robots. Wiley.

S4A (2015). Scratch for Arduino. http: / / s 4 a. cat/. Acessado em 15 de junho de 2015.

Silva, A. A., das Graças Coelho, M., Barros, R. P., and Gonçalves, L. M. (2009). A robótica pedagógica no contexto da educação infantil: auxiliando o alfabetismo. $R e$ vista Tecnologias na Educação, 1(1).

Zilli, S. R. (2004). A robótica educacional no ensino fundamental: Perspectivas e práticas. Master's thesis, Universidade Federal de Santa Catarina. 